\title{
MONUMENTS ET PAYSAGES DE JOHN B. GREENE
}

L'œuvre de John B. Greene se présente comme une floraison étonnante, trop vite interrompue. Si le nom de ce photographe est toujours mentionné au côté de ceux d'autres praticiens voyageurs du milieu du dix-neuvième siècle, ses travaux ont été peu étudiés en tant que tels ${ }^{1}$. Son œuvre s'avère pourtant remarquable par sa qualité technique ; elle est surtout véritablement originale en raison de l'intensité singulière avec laquelle elle condense les tendances et les aspirations coexistant à l'époque - pour les dépasser en un style propre : les vues de Greene sont destinées à servir les progrès de l'archéologie ; elles portent l'empreinte d'une conception romantique des ruines et du paysage; elles font aussi écho aux débats contemporains, relatifs à la photographie et à la peinture; mais elles manifestent, dans le même temps, une certaine hardiesse de sorte qu'elles participent à un renouvellement de la figuration des monuments et des sites.

De nationalité américaine, Greene naît en France en 1832. Il est habité de deux passions : l'archéologie et la photographie ${ }^{2}$. Alors qu'il est trop jeune pour bénéficier d'une

\footnotetext{
${ }^{1}$ Voir cependant Bruno Jammes : «John B. Greene, an American Calotypist » dans History of photography volume 5, number 4, octobre 1981, pp. 305 à 324. Des ouvrages plus généraux apportent des éléments sur son œuvre : Sylvie Aubenas, Jacques Lacarrière, Voyage en Orient, Paris, Bibliothèque nationale de France / Hazan, 1999 ; Claire Bustarret, Parcours entre voir et lire. Les albums de voyage en Orient (1850-1880), thèse soutenue sous la direction d'Anne-Marie Christin, Paris VIII, 1989 ; Nissan N. Perez, Focus East. Early Photography in the Near East. 1839-1885, New York, Harry N. Abrams / Domino, 1988, p. 173. Richard R. Brettell, Paper and Light. The Calotype in France and Geat Britain, 1839-1870, Houston, The Museum of Fine Arts and Chicago, The Art Institute of Chicago, 1984, pp. 173 à 175. Kathleen Stewart Howe, Excursions along the Nile : The Photographic Discovery of Ancient Egypt, Santa Barbara, Santa Barbara Museum of Art, 1994, p. 28. Un article a été consacré à John B. Greene par Will Stapp dans John Hannavy dir., Encyclopedia of Nineteenth-Century Photography, New York / London, Taylor \& Francis, 2008, pp. 619 à 622.

${ }^{2}$ John B. Greene est membre de la «Société Asiatique » qui est tournée vers les études de l'Orient; il est également un des membres fondateurs de la «Société Française de Photographie » en 1854.
} 
investiture officielle, il voyage grâce à l'aisance financière de son père qui est un riche banquier; il se rend en Égypte et en Haute Nubie, en 1853-1854 : il suit le cours du Nil, d'Alexandrie à la seconde cataracte. C'est alors qu'il réalise plus de deux cents calotypes, dont une partie sera réunie, en 1854, dans Le Nil: Monuments - Paysages, Explorations photographiques. En 1855, il est autorisé à effectuer des fouilles dans le temple mortuaire de Ramsès III, à Thèbes. Il réalise des vues de la progression de ses travaux et des sites environnants ; à son retour, il publie une étude des hiéroglyphes de ce site $^{3}$. Il fait encore des fouilles, en 1855-1856, en Algérie où il fait aussi des photographies ${ }^{4}$. Mais, gravement malade, Greene meurt dès 1856, à l'âge de vingt-quatre ans.

\section{La moisson d'un jeune archéologue photographe en Égypte}

La part la plus remarquable du travail de Greene a été réalisée lors de son parcours du Nil, de janvier à mars 1854. Les vues prises sont classées en trois catégories : «Inscriptions », «Paysages » et «Monuments »; elles sont le plus souvent signées en bas, à droite, et numérotées en bas, à gauche : une lettre majuscule, « $\mathrm{M} », \ll \mathrm{P} »$ ou $« \mathrm{I} »$ (assortie d'un numéro) signale la catégorie concernée. Ces indications ont sans doute été portées sur les négatifs puisqu'elles apparaissent en blanc sur les tirages. Un portefeuille in folio, non relié (intitulé Sculptures et Inscriptions égyptiennes) comporte 102 «Inscriptions »; il est conservé à la Bibliothèque de l'Institut de France. Le Nil - Monuments, Paysages, Explorations photographiques (1854) est divisé en deux parties : «Monuments » et « Paysages »; l'album est à la Bibliothèque Nationale de France. Le portefeuille intitulé Monuments et Paysages de la Nubie et de la Haute Égypte, conservé à la Bibliothèque de l'Institut de France ${ }^{5}$, est également séparé en deux parties qui correspondent peu ou prou à celles de l'album de la Bibliothèque nationale, malgré de notables différences ${ }^{6}$.

\footnotetext{
${ }^{3}$ John B. Greene, Fouilles exécutées à Thèbes dans l'année 1855, textes hiéroglyphiques et documents inédits, Paris, Firmin-Didot, 1855. Cet ouvrage, qui comprend 12 planches lithographiées et 12 pages de textes, est à la Bibliothèque Nationale de France; la Bibliothèque de l'Institut de France possède un album du même titre, offert par l'auteur, qui contient également 12 photographies originales.

${ }^{4}$ La Bibliothèque de l'Institut de France contient un album de 14 photographies réalisées en Algérie en 1855 , intitulé Tombeau de la Chrétienne. On ne sait pas où cet album a été imprimé. En Algérie, Greene réalise aussi des photographies de Constantine et des paysages environnants. Ces dernières sont conservées à la National Gallery of Canada, au Metropolitan Museum of Art et au Museum of Modern Art à New York, ainsi qu'au J. Paul Getty Museum à Los Angeles.

${ }^{5}$ Les deux portefeuilles ont été présentés par Greene à l'Académie des Inscriptions et Belles-Lettres, en 1854.

${ }^{6}$ La Société Française de Photographie possède également soixante-dix-huit photographies de monuments et paysages offertes par John B. Greene, le 15 juin 1855 (plus deux épreuves, incluses dans un groupe de photographies données par Blanquart-Évrard).
} 
On sait qu'à partir de 1850, le calotype, plus léger et plus aisé d'utilisation que le daguerréotype, séduit les voyageurs. Exigeant une exposition relativement longue (qui varie en fonction des sujets et des conditions atmosphériques), il convient aux vues de paysage et d'architecture. Ce procédé négatif/positif permet également la réalisation d'albums. Le calotype est surtout employé, entre 1850 à 1860 : ensuite les négatifs sur plaque de verre (qui offrent une plus grande sensibilité) tendent à se généraliser; parallèlement, les centres d'intérêt des voyageurs évoluent vers la figuration des autochtones et des scènes de vie. Greene emploie la technique du «papier ciré sec $»^{7}$ qui a été mise au point par Gustave Le Gray, en 1851 ; ce dernier fait figure d'artiste ${ }^{8}$ et dispense son enseignement à de nombreux photographes (dont le jeune américain ${ }^{9}$ ). Avant d'être exposé, le papier ciré peut être conservé un certain temps; il peut aussi être développé quelques jours après la prise de vue ${ }^{10}$. Si Maxime du Camp n'emploie pas le procédé mis au point par Le Gray ${ }^{11}$, le «papier ciré sec » est utilisé par Félix Teynard, Auguste Salzmann, Greene et bien d'autres voyageurs... Le calotype - quel qu'il soit - tend à éloigner la photographie d'une précision excessive, souvent reprochée au daguerréotype ; l'empreinte pénètre dans la texture fibreuse du papier qui tend à estomper les contours; les dégradés de valeurs sont doux et subtils. Ce procédé semble répondre à la «théorie des sacrifices » qu'un certain nombre de photographes ou de critiques jugent bon d'étendre de la peinture à la photographie, en accord avec l'esthétique exprimée par Baudelaire ou Delacroix ${ }^{12}$.

\footnotetext{
${ }^{7}$ Le papier est ciré avant d'être sensibilisé. Cette technique permet une absorption plus uniforme des préparations chimiques; les papiers ainsi préparés ont une excellente transparence et sont plus raides; ils permettent une plus grande finesse de la représentation. Voir à cet égard Gordon Baldwin, Looking at Photographs. A Guide to Technical Terms, Malibu, J. Paul Getty Museum et London, British Museum, 1991, pp. 84-85.

${ }^{8}$ André Jammes, Eugenia P. Janis, The Art of the French Calotype with a Critical Dictionary of Photographers, Princeton, Princeton University Press, 1983, pp. 38-39. Janet E. Buerger, The Era of the French Calotype, Rochester, International Museum of Photography, 1982, p. 15.

${ }^{9}$ Un cénacle se constitue ; Gustave Le Gray fait avec ses élèves des excursions en forêt de Fontainebleau. Voir à cet égard : Sylvie Aubenas, Gustave Le Gray 1820-1884, Paris, Bibliothèque Nationale de France / Gallimard, 2002 ou Eugenia P. Janis, The Photography of Gustave Le Gray, Chicago, Art Institute and University of Chicago, 1987.

${ }^{10} \mathrm{Le}$ «papier ciré sec » requiert néanmoins un temps d'exposition un peu plus long que le procédé sur papier humide mis au point par Blanquart-Évrard entre 1844 et 1847.

${ }^{11} \mathrm{Il}$ emploie un procédé un procédé sur papier humide.

${ }^{12}$ Baudelaire : «[...] l'art n'étant qu'une abstraction et un sacrifice du détail à l'ensemble, il est important de s'occuper surtout des masses. » dans Salon de 1846, Section III : De la couleur, Paris, Garnier Frères, 1986, p. 107 ; Delacroix, Journal, 13 janvier 1857 : « Sacrifices. Ce qu'il faut sacrifier, grand art que ne connaissent pas les novices. Ils veulent tout montrer. », cité par André Rouillé, Textes et controverses : une anthologie 18161871, Paris, Macula, 1989, p. 97. Gustave Le Gray écrit, quant à lui : « À mon point de vue, la beauté artistique d'une épreuve photographique consiste au contraire presque toujours dans le sacrifice de certains détails, de manière à produire une mise à l'effet qui va quelques fois jusqu'au sublime de l'art. » dans Photographie, traité nouveau, théorique et pratique, 1852, pp. 1-3, cité par André Rouillé dans Textes et controverses: une anthologie 1816-1871, op. cit., p. 99. Francis Wey, un des meilleurs critiques de La Lumière, note : [...] la photographie est très souple, surtout dans la reproduction de la nature; parfois, elle procède par masses,
} 
Les photographies réunies dans Le Nil - Monuments, Paysages, Explorations photographiques ont été tirées, sur papier salé, par l' «imprimerie photographique » de Louis-Désiré Blanquart-Évrard, qui permet - de 1851 à 1855 - la parution de nombreux albums, grâce à une relative industrialisation des tirages ${ }^{13}$. La taille des épreuves positives, obtenues par contact, est déterminée par la taille des négatifs $(240$ x $300 \mathrm{~mm}$, selon le standard des fabricants); il y a cependant une faible déperdition sur les bords, inférieure à 10 $\mathrm{mm}$ ). Comme dans tous les albums photographiques de cette époque, les marges qui entourent les vues collées sont spacieuses. L'ouvrage de Greene est bien plus grand que celui de Maxime Du Camp (paru deux ans plus tôt et qu'il ne pouvait ignorer) ${ }^{14}$; les vues sont présentées dans un format «à l'italienne» (et non en hauteur comme chez Maxime Du Camp). Tout porte à penser que l'ouvrage a été publié à compte d'auteur, en un petit nombre d'exemplaires (on ne sait pas ce qui se serait passé si Greene avait vécu plus longtemps ; le jeune homme envisageait, semble-t-il, une publication chez Goupil ${ }^{15}$ ). L'album qui est à la Bibliothèque Nationale de France diffère du portefeuille qui est à la Bibliothèque de l'Institut (même si tous deux sont divisés en deux parties analogues : «Monuments » et «Paysages ») : le premier ne possède pas de texte, alors que le second comporte une table et des légendes manuscrites; certains paysages ne revêtant pas d'intérêt archéologique se trouvent exclus du portefeuille qui est à l'Institut ${ }^{16}$. Au Musée d'Orsay, sont conservés les classeurs de négatifs utilisés par l'archéologue, au cours de ses deux voyages en Égypte ; ces

\footnotetext{
dédaignant le détail comme un maître habile, justifiant la théorie des sacrifices, et donnant ici l'avantage à la forme, et là aux oppositions de tons. » dans « De l'influence de l'héliographie sur les Beaux-Arts, La Lumière, 9 février $1851, \mathrm{n}^{\circ} 1$, pp. 2-3.

${ }^{13}$ Blanquart-Évrard a mis au point un procédé de tirage plus rapide, par développement à l'acide gallique d'un papier ioduré. Parfois il tirait les planches et publiait l'album. D'autres fois, les planches, tirées dans son imprimerie, étaient éditées ailleurs; c'est par exemple le cas pour Égypte, Nubie, Palestine et Syrie : dessins photographiques recueillis pendant les années 1849, 1850 et 1851, accompagnés d'un texte explicatif de Maxime Du Camp ou Jérusalem. Étude et reproduction photographique des monuments de la Ville Sainte depuis l'époque judäque jusqu'à nos jours d'Auguste Salzmann qui paraissent chez Gide \& Baudry, respectivement en 1852 et en 1856. L'agencement et le choix des vues pouvaient quelques fois résulter du choix des souscripteurs. Ces usages expliquent une disparité entre les différents exemplaires d'albums qui peuvent être consultés aujourd'hui. Le terme d'imprimerie est, à la vérité, impropre pour désigner l'entreprise de Blanquart-Évrard, puisque les photographies originales sont collées dans les albums, qui restent des objets luxueux destinés à une élite. Voir Isabelle Jammes, Blanquart-Évrard et les origines de l'édition photographique française. Catalogue raisonné des albums photographiques édités, Genève, Droz, 1981.

${ }^{14}$ L'album de Du Camp fait 440 x $310 \mathrm{~mm}$, celui de Greene 390 x $550 \mathrm{~mm}$.

${ }^{15}$ Voir l'article de Will Stapp dans Encyclopedia of Nineteenth-Century Photography, art. cit., p. 621.

${ }^{16} \mathrm{~L}$ 'album de la Bibliothèque nationale compte 48 planches de Monuments et 46 planches de Paysages; le portefeuille de la Bibliothèque de l'Institut comporte 42 planches de Monuments et 41 planches de Paysages.
} 
classeurs où figurent le lieu et la date des prises de vue permettent de reconstituer l'itinéraire du jeune homme ${ }^{17}$.

Lorsque Greene se rend sur les bords du Nil, il s'inscrit bien évidemment dans un mouvement d'intérêt beaucoup plus large pour ces contrées. Alors qu'au dix-huitième siècle, les étapes de tout voyage vers le Levant étaient l'Italie et la Grèce, au dix-neuvième siècle, le champ d'investigation s'étend vers le Proche-Orient, et notamment vers l'Égypte. La fascination pour ce pays correspond à un désir de retrouver l'enfance du monde, l'origine de la civilisation. Les voyages orientaux de photographes tels que Maxime Du Camp, Félix Teynard $^{18}$ ou Greene sont les avatars de ces tendances plus générales. Toutefois au rêve entretenu par la littérature ou la peinture, la photographie oppose - dans une certaine mesure une réalité plus contingente. En matière de reproduction de monuments orientaux, il y a alors une forme de consensus sur le statut documentaire de la photographie, chargée de rapporter des informations scientifiques. À propos de l'album de Maxime Du Camp, Louis de Cornemin note en 1852 : «Nous n'avons plus besoin de monter sur le vaisseau des Cook et des Lapeyrouse pour tenter de périlleux voyages; l'héliographie, confiée à quelques intrépides, fera pour nous le tour du monde, et nous rapportera l'univers en portefeuille, sans que nous quittions notre fauteuil. ${ }^{19}$. Les images réalisées en Orient, dans les années 1850, bénéficient d'une heureuse légitimité en tant qu'archives du monde. Les albums successifs, qui se complètent et se répondent, manifestent l'utopie d'une forme d'inventaire méthodique de réalités lointaines ${ }^{20}$; ils s'apparentent aux vastes recueils et aux collections systématiques dont les hommes du dix-neuvième siècle sont friands.

Les vues de Maxime Du Camp, frontales et impersonnelles, manifestent tout particulièrement cette volonté documentaire. Les cadrages des photographies d'Auguste Salzmann sont également liés à des nécessités scientifiques : ce photographe se rend à Jérusalem pour recueillir les preuves photographiques des théories alors controversées d'un

\footnotetext{
${ }^{17}$ Claire Bustarret, Parcours entre voir et lire. Les albums de voyage en Orient (1850-1880), op. cit., tome 2, pp. 162 à 170 et pp. 306-307. Au musée d'Orsay sont également conservés des tirages de Greene inclus par Théodule Devéria dans sa vaste collection iconographique.

${ }^{18}$ Félix Teynard, Égypte et Nubie, sites et monuments, Paris, Goupil, 1858.

${ }^{19}$ Louis de Cornemin, «À propos d'Égypte, Nubie, Palestine et Syrie, de Maxime Du Camp » dans La Lumière $\mathrm{n}^{\circ} 25,12$ juin 1852 , p. 98.

${ }^{20}$ Claire Bustarret, Parcours entre voir et lire. Les albums de voyage en Orient (1850-1880), op. cit., pp. 190 à 193.
} 
archéologue, Félix-Joseph Caignart de Saulcy ${ }^{21}$. Au moment où se développe l'intérêt pour le passé, les vues rapportées par les photographes voyageurs qui se rendent en Orient sont vouées à servir le travail des historiens. Les vues de Greene manifestent avec évidence ce souci de fournir des informations utiles et pertinentes. Lorsqu'il ne peut photographier les inscriptions en raison du manque de lumière, il réalise des «estampages », clichés d'empreintes séchées (en papier mâché) des reliefs gravés dans la pierre ${ }^{22}$; cette méthode manifeste bien la dimension scientifique de l'entreprise du jeune homme. Dans les classeurs conservés au Musée d'Orsay, le nombre important des négatifs appartenant à la catégorie des «Inscriptions » signale le sérieux de la démarche égyptologique de Greene. Les vues de fouilles de 1855 et 1856 témoignent encore de l'intense fascination de l'archéologue pour ses recherches.

Cependant les photographies des monuments anciens font aussi écho aux réflexions des hommes de lettres et des critiques d'art. Dès 1767, Diderot notait à propos des peintures de ruines : «On n'est arrêté que par l'idée de la puissance éclipsée des peuples qui ont élevé de pareils édifices. Ce n'est pas de la magie du pinceau, c'est des ravages du temps que l'on s'entretient $[\ldots] »^{23}$. En raison des textes nombreux qu'elles convient, mais également grâce à leurs traits propres, les épreuves photographiques exaltent le sentiment de l'irréversibilité de la fuite du temps. Les images des édifices sont vides d'êtres animés; alors que Maxime Du Camp faisait parfois poser un nubien de son équipage afin de donner l'échelle, la figure humaine est quasiment absente des vues de Greene ${ }^{24}$. Cette évacuation aboutit à une exclusive polarisation sur les pierres et le moment historique glorieux avec lequel elles établissent un

\footnotetext{
${ }^{21}$ Félicien-Joseph Caignart de Saulcy, «Exploration photographique de Jérusalem par M. Auguste Salzmann » dans Le Constitutionnel, 24 mars 1855, pp. 2-3: « Je voudrais [...] essayer de montrer quels secours la science peut recevoir de la Photographie [...]. M. Auguste Salzmann, frappé de mon opiniâtreté à soutenir que j'avais bien vu les faits que l'on me contestait, s'est chargé d'aller vérifier sur place toutes mes assertions, à l'aide d'un dessinateur fort habile, en vérité, et dont il est difficile de suspecter la bonne foi, c'est-à-dire le soleil, qui n'a d'autre parti pris que celui de reproduire ce qui est. ».

${ }^{22}$ Lorsque Greene exposa sa technique, il y eut un malentendu : certains crurent qu'il avait inventé un nouveau procédé permettant de photographier dans l'obscurité (voir La Lumière $\mathrm{n}^{\circ} 27$ du 8 juillet 1854, p. 105). Le jeune homme dut opérer une clarification (La Lumière ${ }^{\circ} 29$ du 22 juillet 1854, p. 115) : «Dans votre dernier numéro, vous annoncez que j'ai découvert le moyen de faire des épreuves photographiques dans les lieux obscurs. Je n'ai jamais eu cette prétention. J'ai seulement dit que j'avais le moyen de reproduire, par une nouvelle application de la photographie, les sculptures et impressions des intérieurs, quelles que fussent les conditions d'éclairage et de position. Cette dernière condition excluait l'emploi immédiat de la chambre noire. L'application dont je parle est la reproduction des estampages. ». Les « estampages » sont conservés au Musée d'Orsay.

${ }^{23}$ Diderot, Ruines et paysages. Salon de 1767, Paris, Hermann, 1995, p. 335.

${ }^{24}$ Quelques rares épreuves font exception. L'une d'elle montre une sorte de case bâtie le long du Nil, dans laquelle une silhouette floue est discernable ; on peut penser qu'il s'agit d'un compagnon de voyage du jeune photographe.
} 
trait d'union. Selon le paradigme indiciaire décrit par Carlo Ginzburg ${ }^{25}$, les traces photochimiques de restes des civilisations disparues invitent le spectateur à une forme de reconstruction du passé.

\section{La ruine, comme modulateur de lumière}

Dans le Bulletin de la Société Française de Photographie ${ }^{26}$, il est rapporté que, pour Greene, l'éclairage le plus propice à la prise de vue est celui du matin (entre 7 heures et 11 heures) ou de l'après-midi (après 15 heures). La clarté singulière des pays orientaux a impressionné la plupart des voyageurs - qu'ils soient peintres ou hommes de lettres. Toutefois la pratique de la photographie ne pouvait que renforcer l'attention portée, de manière concrète, aux changements atmosphériques et aux variations lumineuses. Les travaux de Félix Teynard, d'Auguste Salzmann ou encore d'Henry Cammas ${ }^{27}$ en attestent. Mais Greene a tout particulièrement su jouer - à la fois avec vigueur et subtilité - de la circulation de la lumière sur les volumes des édifices afin de créer des effets plastiques et sémantiques.

L'éclairage latéral tend à exalter la disposition et les formes des monuments. Le sphinx de Gizeh étire sur le sable son ombre allongée, tandis qu'un de ses profils baigne dans une pénombre tamisée. Un important contraste de valeur souligne la disposition angulaire des deux faces visibles de la pyramide de Chéops. Sur une vue du temple de Gournah, l'ombre portée de la couverture du péristyle plonge dans l'obscurité la partie supérieure du mur du fond tandis que, plus bas, la réplique sombre de chaque colonne crée un rythme complexe.

La célébration des formes architecturales par les jeux de la lumière n'est pas neuve. Les peintres en ont usé ; la parenté frappe surtout avec l'estampe qui, comme la photographie, exploite un dégradé monochrome de tonalités pour flatter les volumes et les proportions des bâtiments. Les eaux-fortes de Piranèse reviennent tout particulièrement à l'esprit: dans les œuvres de cet artiste, amoureux d'architecture et d'archéologie - que ne pouvait manquer de connaître le jeune Greene - l'éclairage se fait quasiment lyrique afin de permettre un éloge

25 Carlo Ginzburg, «TRACES. Racines d'un paradigme indiciaire » dans Mythes, Emblèmes, Traces Morphologie et Histoire, Paris, Flammarion, « Nouvelle Bibliothèque Scientifique », 1989, pp. 139 à 180.

${ }^{26}$ «Assemblée générale de la société. Procès verbal de la séance du 15 juin 1855 » dans Bulletin de la Société Française de Photographie, Première année, n 6, juin 1855, p. 165.

${ }^{27}$ Henry Cammas, L'Égypte photographiée, Maison Lafitte, Henry Cammas, 1864. 
passionné des ruines romaines et de suggérer une forme de renaissance du modèle antique ${ }^{28}$. On pense également aux lithographies figurant dans les Voyages pittoresques qui se développent dans la première moitié du dix-neuvième siècle ; là aussi, les ressources des jeux de lumière sont exploitées pour mettre en valeur les édifices. Cependant, dans ces ouvrages, le souci d'embellissement poétique, la recherche du pittoresque ${ }^{29}$, la volonté d'animer les sites par la présence de petits personnages ${ }^{30}$ ont tendance à détourner l'attention de l'observateur des seuls volumes architecturaux. Peu à peu, d'ailleurs, les archéologues reprocheront à ces représentations leur manque de fidélité ; après 1850, la photographie tend à remplacer le procédé lithographique : «Le genre disparaît alors avec la matière qui avait assuré sa propagation et son succès ; ainsi se vérifie une fois de plus l'idée qu'un certain sentiment est généralement lié à une certaine technique, et que la disparition de l'une doit entraîner celle de 1'autre. $»^{31}$. De fait, la photographie conduit assez naturellement à plus d'exactitude et - dans un premier temps - évacue presque la figuration de la vie humaine.

De façon plus générale, on peut dire que l'ordonnancement complexe de l'édifice architectural s'offre à la circulation de la lumière qui vient investir les volumes et les trouées du bâti : la clarté caresse et flatte les formes ; elle magnifie les textures et les granulosités ; des pans de la construction font obstacle au flux photonique, générant des ombres portées dont la forme peut d'ailleurs être modifiée par les irrégularités du fond sur lequel elles tombent ; des éléments architecturaux que l'on ne voit pas sont parfois suggérés par l'ombre qu'ils projettent au sol; des parois peuvent aussi jouer le rôle de réflecteurs, renvoyant la luminosité en un écho diffus sur les volumes alentours. Chateaubriand chante les «formes ouvertes» des ruines qui favorisent sans doute, plus que d'autres édifices, les «jeux de lumière $»^{32}$.

\footnotetext{
${ }^{28}$ Didier Laroque, Le Discours de Piranèse. L'ornement sublime et le suspens de l'architecture, Paris, Éditions de la Passion, 1999.

${ }^{29}$ Au sens où l'entend William Gilpin, « On Picturesque Beauty » dans Three Essays on Picturesque Beauty [1794], London, T. Caldwell and W. Davies, 1808.

${ }^{30} \mathrm{La}$ réalisation de ce type d'ouvrages engage plusieurs personnes : les voyageurs qui réalisent des croquis rapides sur place, les lithographes de métier qui transforment ces premières esquisses pour en faire des œuvres achevées et les éditeurs ; parfois aussi sont requis les services de « faiseurs de bonshommes».

${ }^{31}$ Jean Adhémar, La France romantique. Les lithographies de paysage au XIXe siècle, Paris, Somogy éditions d'art, 1997, pp. 106-109.

${ }^{32}$ Chateaubriand, Génie du christianisme dans Essai sur les révolutions. Génie du christianisme, texte établi, présenté et annoté par Maurice Regard, Paris, Gallimard, «Bibliothèque de la Pléiade », 1978, livre IV, chap. IV, pp. 883 à 885.
} 
De 1922 à 1930, László Moholy-Nagy travaille à la réalisation d'un «modulateur de lumière », sculpture cinétique et lumineuse, susceptible d'orchestrer des variations d'ombres et des reflets changeants sur les murs environnants ${ }^{33}$. Comme le «modulateur » de l'artiste hongrois, l'édifice architectural - et davantage encore la ruine - exploite la circulation liquide de la lumière au sein de formes trouées ; cependant la versatilité des effets ne vient pas, dans ce cas, de la mobilité de la structure, mais des changements d'orientation et de qualité de l'éclairage. Bien après Greene et ses contemporains, des photographes ont exploité cette aptitude des bâtiments à «moduler» la lumière. Qu'il s'agisse de tenants du modernisme (Herbert List, Charles Sheeler, Werner Mantz), de chantres de l'architecture du troisième Reich (Max Baur), de praticiens collaborant étroitement avec des architectes (Lucien Hervé avec Le Corbusier) ou encore de contemporains qui font de la prise de vue l'instrument d'une réflexion critique sur l'architecture (Guy Dépollier) ${ }^{34}$, des photographes tirent parti - à l'instar de Greene (et d'autres photographes de son temps) - de l'aptitude du flux lumineux à investir les formes complexes des édifices, comme l'air investit les colonnes d'un orgue pour créer des sons.

Dans les vues de monuments réalisées par Greene, les contrastes sont importants : certaines zones sont d'un noir profond, tandis que d'autres confinent au blanc. La présence d'ombres accusées tend à intensifier le sentiment de l'éclat de la lumière ; lorsqu'il passe de zones très sombres à des zones claires, l'observateur éprouve une sensation que l'on peut rapprocher de celle de l'éblouissement. Mais le jeune photographe exploite également, avec une virtuosité et une sensualité particulières, les possibilités du calotype qui permet de subtils dégradés de valeurs. Il évite toute surcharge et obtient des ombres veloutées et tamisées. Dans une photographie représentant une statue de femme à Spéos de Phré (Ibsamboul), l'ombre conduit, à travers des modulations infinies, du noir complet jusqu'à l'extrême clarté. La lumière liquide épouse la pierre, dont elle exalte la granulation et les volumes ; elle flatte amoureusement les détails et le relief des formes sculptées. La variation sensuelle de l'ombre, qui va s'éclaircissant, anime les volumes de pierre, qui acquièrent ainsi une présence tangible (illustration).

\footnotetext{
${ }^{33}$ László Moholy-Nagy, Peinture Photographie Film et autres écrits sur la photographie, Paris, Gallimard, « Folio Essais », 2007, pp. 57 à 59.

${ }^{34}$ Vues d'architecture, catalogue d'une exposition qui s'est tenue au Musée de Grenoble, Paris-Grenoble, RMN / Musée de Grenoble, 2002, pp. 38, 69, 94, 138-139, 167 ; Robert Elwall, Building with Light. The International History of Architectural Photography, London, Royal Institute of British Architects / Merrell Publishers, 2004, pp. 103, 131, 145, 174.
} 
Souvent, chez Greene, les formes semblent sortir de l'obscurité pour advenir au regard. Le jeu des ombres et des lumières opère une dramatisation de l'apparition des édifices: «Les pylônes égyptiens, les hiéroglyphes, les statues encore ensablées semblent sortir du néant, de l'ombre, et se laisser gagner - pour être vues enfin - par le soleil. ${ }^{35}$. Le dynamisme induit par le crescendo de la lumière magnifie les formes architecturales et, en même temps, théâtralise une réapparition métaphorique du passé : c'est de l'obscurité des temps anciens que les édifices émergent pour s'offrir au regard. L'incomplétude de la perception se fait également, pour le spectateur, aiguillon du désir de reconstruction du passé (d'autant que l'incomplétude due à l'ombre s'ajoute au caractère fragmentaire de la ruine, mangée par l'usure et pour partie encore enfouie sous les sables).

Un dégradé sensuel peut également être observé dans une vue représentant les stèles de Silsilis : l'ombre portée de l'entablement, sur lequel tombe une lumière dense, engendre la bipartition du mur du fond entre une zone sombre, frangée, et une partie claire. L'ombre n'apparaît plus comme l'antithèse de la lumière, mais bien comme un de ses avatars ; elle la prolonge en quelque sorte, s'en fait l'écho et rend ainsi perceptible la fluidité, dans l'espace occupé par les volumes architecturaux, du rayonnement photonique - dont on ne peut oublier par ailleurs qu'il a permis la prise de vue. L'organisation des ombres et des lumières est marquée de précarité; liée à la position de l'astre solaire et aux transformations atmosphériques, elle prête vie et actualité aux édifices immémoriaux.

L'attention portée à la lumière par les premiers photographes voyageurs est, pour partie, conditionnée par la technique même qu'ils utilisent. La photographie sur papier correspond (même si elle fut ensuite remplacée par d'autres procédés) à un artisanat abouti ${ }^{36}$. La réalisation du calotype inclut bon nombre d'étapes : minutieuse préparation chimique du papier selon des dosages susceptibles de variantes selon les praticiens, exposition dans la chambre noire, révélation, fixation, puis plus tard obtention d'un positif par $\operatorname{contact}^{37}$. La prise de vue ne représente donc qu'un moment dans un processus long, qui génère une forte conscience du phénomène de la photosensibilité. Pour le calotypiste qui possède nécessairement des rudiments en chimie, le monde apparaît peu ou prou comme un réflecteur

\footnotetext{
${ }^{35}$ Michel Frizot dir., Nouvelle histoire de la photographie, Paris, Adam Biro/Bordas, 1994, p. 79.

${ }^{36}$ Eugenia P. Janis, André Jammes, The Art of French Calotype with a Critical Dictionary of Photographers : 1845-1870, op. cit.

37 Luis Nadeau, Encyclopedia of Printing, Photographic and Photomechanical Processes, volume 1, Fredericton, Atelier Luis Nadeau, 1989, p. 52.
} 
du rayonnement lumineux ${ }^{38}$. La chambre noire symbolise en outre l'obligation d'une forme de passage de la clarté par l'obscurité pour pouvoir se manifester à nouveau. La nécessité de l'étape du négatif s'accompagne de l'idée d'une réversibilité de l'ombre et de la lumière. La calotypie se présente donc comme une forme d'artisanat de lumière. À propos de la Digue au bord du Nil de Léon Belly, Théophile Gautier note, en 1859 : «La lumière, arrivée au plus au point d'intensité, brûle les couleurs comme dans les épreuves photographiques $[\ldots] »^{39}$; ce commentaire témoigne du fait que la photographie se donne alors comme le paradigme d'une action transformatrice de la lumière.

Parfois, des ombres masquent certaines parties des édifices. Sur une vue de l'entrée du mur sud de la salle hypostyle de Karnak, le tour intérieur de la porte est cerné d'un noir dense, correspondant à l'ombre propre du bâti ; l'ombre portée crée, quant à elle, une tâche allongée, horizontale, qui vient quasiment fermer un cerne rectangulaire très foncé - dont le dessin fait écho à la forme même de la photographie. L'obscurité oblitère presque complètement, par endroits, les détails. La répartition des ombres et des lumières entraîne dès lors une reconfiguration de l'apparence de l'édifice (illustration). Au dessein de l'architecte et au burin du sculpteur succède le ciseau du soleil. En certains endroits, les volumes de pierre se trouvent nouvellement évidés. La densité extrême de certaines ombres amorce même, par places, un déni de la troisième dimension. L'ombre n'est plus un fond, mais une tache ; elle s'impose comme un temps fort de la composition. De telles vues préfigurent une esthétique picturale qui un peu plus tard osera des ombres violentes, que ce soit dans le champ de la peinture orientaliste ${ }^{40}$, pour la représentation de monuments (on pense évidemment à certaines toiles de Claude Monet $)^{41}$ ou encore pour la figuration de tout autre sujet ${ }^{42}$.

\section{L'attrait des étendues infinies et des terres rocailleuses}

Les albums de Maxime Du Camp ou d'Auguste Salzmann comportent peu de sites naturels, exempts de toute ruine. Félix Teynard s'intéresse davantage aux paysages, en eux-

\footnotetext{
${ }^{38}$ Selon Michel Frizot, le calotype «instaure avec son opérateur, le photographe, une relation de fascination et d'extase à l'égard de l'objet, de l'espace, de la lumière [...]»: «La parole des primitifs. À propos des calotypistes français » dans Études photographiques n 3, Novembre 1997, p. 45.

39 Théophile Gautier, Exposition de 1859, Heidelberg, C. Winter, 1992, p. 46.

${ }^{40}$ Voir Christine Peltre, Les Orientalistes, Paris, Hazan, 1997, p. 173.

${ }^{41}$ Voir par exemple : «La Cathédrale de Rouen. Le portail et la tour d'Albane, plein soleil. Harmonie bleu et or », 1894, toile 1,07 x 0,73 m, Musée du Jeu de Paume. Les ombres accusées retiennent presque davantage l'attention que les volumes de pierre.

${ }^{42}$ Claude Monnet, « Meule en hiver », 1891, toile 0,66 x 0,90 m, Boston, Museum of Fine Arts.
} 
mêmes. Mais ces derniers sont bien plus nombreux encore chez Greene. Le fait que Le Nil: Monuments - Paysages, Explorations photographiques soit divisé en deux parties - dont l'une consacrée aux paysages - signe l'importance que le jeune photographe accorde à cette catégorie (privée de toute utilité archéologique). Quand il n'y a pas de légende ${ }^{43}$ et que tout édifice reconnaissable fait défaut, il peut être difficile de supputer la localisation précise de la prise de vue, voire même parfois d'inférer l'échelle exacte de la représentation.

Greene a suivi l'enseignement de Gustave Le Gray, qui s'adonne à la photographie de paysage ; il a participé avec ce dernier à des excursions en forêt de Fontainebleau, où il a luimême réalisé des vues de hautes futaies et de feuillages en 1852 : par son rendu moelleux, par la variété de ses nuances et sa finesse, le «papier ciré sec »sert la transcription de la lumière vibrant au travers du rideau des branches et des feuilles ${ }^{44}$. Dans la forêt de Fontainebleau, les praticiens côtoient les peintres de Barbizon. L'attrait des photographes pour les lieux champêtres croise une évolution générale du goût qui valorise de plus en plus la peinture sur le motif et la figuration de la nature.

Progressivement, les peintres orientalistes se tournent également davantage vers le paysage $^{45}:$ «Le Nil est plus grand peut-être, quand il coule dans la solitude, entre ses rives couvertes de bois de palmiers et de sycomores ou au milieu des sables du désert, que quand il passe au pied des villes, avec ses barques flottantes et la joyeuse population de ses bords. ${ }^{46}$, confie Narcisse Berchère. Cependant, «[...] en cette époque où les peintres-voyageurs n'en sont pas avares, les mots sont en avance sur l'exécution. ${ }^{47}$; les tableaux achevés restent en général peuplés de personnages typiques et de motifs qui tendent à conférer à l'Orient plus de traits orientaux qu'il n'en possède. Cette avance des mots coïncide peut-être avec celle de la photographie, telle qu'elle est pratiquée par Greene.

La végétation est relativement présente dans les paysages réalisés par le jeune homme - comme d'ailleurs chez Félix Teynard ${ }^{48}$; les palmiers forment des bosquets au bord du Nil ou lancent leurs silhouettes graciles sur les ciels vacants, au-dessus d'étendues arides et

\footnotetext{
${ }^{43}$ L'album conservé à la Bibliothèque nationale de France ne comporte aucun texte.

${ }^{44}$ John B. Greene, «Forêt de Fontainebleau, 1852 » dans Sylvie Aubenas, Gustave Le Gray 1820-1884, op. cit., p. 45.

${ }^{45}$ Christine Peltre, L'Atelier du voyage, Paris, Gallimard, « Le promeneur », 1995, p. 67.

${ }^{46}$ Narcisse Berchère, Le Désert de Suez. Cinq mois dans l'isthme, Paris, Hetzel, 1863, p. 138.

${ }^{47}$ Christine Peltre, Les Orientalistes, op. cit., p. 154.

${ }^{48}$ Kathleen Stewart-Howe, Félix Teynard, calotypes of Egypt : a catalog raisonné, New York, Hans P. Kraus, 1992.
} 
caillouteuses. L'arbre constitue un des accessoires classiques de la peinture de paysage ; les forêts et les éléments botaniques sont très présents chez les photographes de la «tradition française du calotype » qui ont certainement influencé Greene ${ }^{49}$; le palmier constitue aussi indéniablement un «opérateur » d'exotisme. Toutefois, chez Greene, les troncs ont tendance à s'espacer; le jeune photographe «joue en géomètre d'un point de repère, palmier, groupe d'arbres, monument lointain, pour structurer une image pleine du vide du ciel, du sable et de l'eau. $»^{50}$. Ses vues, parfois minimales, manifestent une grande rigueur de composition et une exceptionnelle qualité graphique.

Mais surtout le jeune praticien n'hésite pas à prendre du recul et à ménager dans ses images d'importants espaces de respiration. Les zones vacantes sont dès lors aussi importantes que les éléments pleins. Les clichés, qui embrassent l'immensité des ciels et des sables, rendent sensible l'étendue qui excède les choses. Parfois, le reflet des berges dans les eaux du Nil suggère la circulation de la lumière ; le léger flou des palmes d'un dattier peut renvoyer au mouvement de l'air. La texture granuleuse du papier entraîne une perte de détails, de sorte que le territoire paraît baigner dans une vapeur impalpable : la sensation d'une continuité spatiale prime sur la circonscription précise des éléments représentés. Au vu de ces images, on comprend aisément qu'un critique tel que Francis Wey, subjugué par le génie de Delacroix et fervent défenseur du calotype, ait eu tendance à placer cette technique du côté de l'art des coloristes, à l'opposé de celui des partisans de la ligne. La modulation veloutée des tons transcrit les variations subtiles de l'air qui baigne les choses. Une gamme de valeurs très étendue traduit l'étagement des plans dans la profondeur du champ; les formes s'estompent progressivement pour se perdre au loin dans le grain du papier: l'horizon ne paraît pas constituer une limite, mais une ouverture sur l'infini ${ }^{51}$.

Chez Greene, le cadrage est parfois très large de sorte que les ruines, de petite taille, se perdent dans le paysage; les vues suggèrent dès lors les étendues incommensurables et abandonnées, qui s'étendent au-delà des limites du champ ; au dix-neuvième siècle, le désert est associé au sentiment du sublime, par son immensité qui éveille l'idée de l'infini, son aspect informe et chaotique, sa vacuité. Au sujet du désert, Vivant Denon écrit : «[...] nom terrible à qui l'a vu une fois, horizon sans bornes, dont l'espace vous oppresse, [...] où la

\footnotetext{
${ }^{49}$ Bruno Jammes, «John B. Greene, an American Calotypist », art. cit., p. 316.

${ }^{50}$ Sylvie Aubenas, Voyage en Orient, op. cit., pp. 28-29.

${ }^{51}$ Michel Collot, L'Horizon fabuleux, tome 1: XIX ${ }^{\mathrm{e}}$ siècle, Paris, Corti, 1988, p. 46 : l'auteur montre qu'au moment du romantisme, l'horizon ne borne plus le site, mais caractérise une illimitation du paysage.
} 
colline ne vous cache ou ne vous découvre que la décrépitude et la décomposition, où le silence de la non-existence règne seul sur l'immensité. $\gg^{52}$. Quant à Chateaubriand, il confie : «Quand on voyage dans la Judée, d'abord un grand ennui saisit le cœur; mais lorsque, passant de solitude en solitude, l'espace s'étend sans bornes devant nous, peu à peu l'ennui se dissipe, on éprouve une terreur secrète, qui, loin d'abaisser l'âme, donne du courage, et élève le génie. ${ }^{53}$. Pour les romantiques dont Greene reçoit l'héritage, le paysage désertique se présente non comme un objet extérieur mais comme une expérience susceptible de hausser le sujet percevant vers des considérations métaphysiques ${ }^{54}$. Les vues, vides de toute vie, suggèrent le vertige éprouvé devant un espace et un temps qui paraissent inconcevables. Elles rendent sensible la façon dont le paysage investit l'être qui le contemple, dont le site se mue en rêverie silencieuse. Elles suggèrent un état psychique qui, bien que naissant chez le sujet percevant, est intrinsèquement liée au lieu - on pense dès lors à la «Stimmung » que définit Georg Simmel ${ }^{55}$.

Les constituants du site ont tendance à se noyer dans la texture grenue du papier ; ils semblent baigner dans un voile atmosphérique; les contours se diluent et l'espace paraît continu et indivisible, ouvert sur l'extérieur. Les cieux vides et blafards occupent parfois plus des deux tiers de la représentation. Au loin, l'horizon aimante le regard ; mais il n'y a pas de lignes de fuite fortes marquant la perspective et assignant à l'observateur une place précise. Le flou, souvent ménagé au premier plan, donne plutôt au spectateur une sensation de flottement et de légère instabilité. Sans doute de telles représentations ont-elles pu interpeller des peintres tournés vers le questionnement de la construction traditionnelle de l'espace pictural.

Eugène Fromentin, évoquant le désert, parle d' « [...] une grande chose sans forme, presque sans couleur, le rien, le vide et comme un oubli du bon Dieu; des lignes fuyantes, des ondulations indécises [...] ${ }^{56}$. Chez Greene, des étendues vagues de cailloux et de sable

\footnotetext{
${ }^{52}$ Vivant Denon, Voyage dans la Basse et la Haute Égypte pendant les campagnes du général Bonaparte [1802], Paris, Éditions Pygmalion / Gérard Watelet, 1990, p. 141.

${ }^{53}$ Chateaubriand, Itinéraire de Paris à Jérusalem dans Euvres romanesques et voyages, tome 2, texte établi, présenté et annoté par Maurice Regard, Paris, Gallimard, «Bibliothèque de la Pléiade », 1969, p. 999.

${ }^{54}$ Yvon Le Scanff, Le Paysage romantique et l'expérience du sublime, Seyssel, Champ Vallon, «Pays / Paysages », 2007, p. 142.

${ }^{55}$ Georg Simmel, « Philosophie du paysage » [1913] dans La Tragédie de la culture et autres essais, Paris, Petite Bibliothèque Rivages, 1988, pp. 229 à 243.

${ }^{56}$ Eugène Fromentin, Un Été dans le Sahara [1857], texte établi et présenté par Anne-Marie Christin, Paris, Éditions du Sycomore, 1981, p. 95.
} 
s'étendent sous les cieux immenses (illustration). Une attention particulière est portée à la texture des sols, au grain du terrain rocheux qui constitue parfois l'unique sujet de la prise de vue $^{57}$; la substance même de la croûte terrestre prend là toute son importance. Dans son originalité, le travail de Greene rejoint et accompagne les aspirations qui se manifestent (de façon timide encore) chez certains peintres orientalistes contemporains, emportés par un désir de prise en compte accrue de la perception des choses. Une propension à affronter un réel « réduit aux mouvements et à la texture des sols » ${ }^{58}$ peut parfois être constatée $:$ il est arrivé à Narcisse Berchère ${ }^{59}$ ou à Léon Belly ${ }^{60}$ de montrer des chaos de sables et de roches, vides de tout élément anecdotique; Eugène Fromentin a fait des mines de plomb ${ }^{61}$ ou encore des esquisses à 1 'huile ${ }^{62}$ avec «presque rien » (même si ses œuvres abouties restent tributaires d'une image plus traditionnelle de l'Orient).

Les travaux de Greene doivent être remarqués pour l'acuité singulière avec laquelle ils croisent les tendances et les aspirations d'une époque. Héritière de l'esthétique romantique, son œuvre reçoit l'influence des estampes de monuments et d'architecture; elle est à même d'éveiller le sentiment du sublime; elle montre magistralement la façon dont le calotype permet le sacrifice du détail pour privilégier une harmonie générale, renvoyant ainsi aux débats qui animent à l'époque la critique spécialisée. Dans le même temps, elle fournit des renseignements archéologiques précieux et participe à une vaste entreprise scientifique de collection d'informations nécessaires aux historiens. Mais elle manifeste aussi une audace tout à fait singulière : elle préfigure ainsi la manière dont les ombres et les lumières, les pleins et les vides, l'espace de la représentation, le grain et la matière pourront être traités par la suite dans la peinture occidentale. Les vues de Greene montrent bien combien toutes les tendances - à la croisée desquelles elles se situent - s'entretissent sans s'opposer les unes aux autres. Elles travaillent, chez lui, à créer un style personnel, une vision qui est alors sans équivalent.

\section{Danièle Méaux}

\footnotetext{
${ }^{57}$ Martin Parr, Gerry Badger, The Photobook : A History (volume 1), Londres, Phaidon, 2004, p. 24.

${ }^{58}$ Christine Peltre, Les Orientalistes, op. cit., p. 77.

${ }^{59}$ Narcisse Berchère, « Après le Simoun », 1864.

${ }^{60}$ Léon Belly, «La mer morte », 1866 ou «Le désert de Nassoub (Sinaï) », 1857.

61 James Thompson, Barbara Wright, Les Orientalistes. Eugène Fromentin, Paris, ACR Éditions, 1987, pp. 5859.

${ }^{62}$ Ibid., pp. 122-123. Par exemple : «Laghouat, 20 juin, 9 heures » (1853). Barbara Wright note d'ailleurs que la familiarité de Fromentin avec la photographie a pu influencer sa manière de peindre.
} 